\title{
Traumatic pseudoaneurysm of aorta: a story of survival
}

\author{
Smeet Patel*, Sanjay Patel, Lakshman Khiria
}

Mayflower Women's Hospital, Ahmedabad, Gujarat, India

Received: 19 January 2022

Accepted: 05 February 2022

\section{*Correspondence:}

Dr. Smeet Patel,

E-mail: smeet@mayflowerhospital.com

Copyright: ( $)$ the author(s), publisher and licensee Medip Academy. This is an open-access article distributed under the terms of the Creative Commons Attribution Non-Commercial License, which permits unrestricted non-commercial use, distribution, and reproduction in any medium, provided the original work is properly cited.

\section{ABSTRACT}

Pseudoaneurysms caused by injury to the abdominal aorta are uncommon, but they are frequently observed after forceful trauma or injury to penetrating arteries. Aortic pseudoaneurysm (AAP) symptoms range from asymptomatic to compression symptoms to severe rupture culminating in mortality. We present a case of a young female who had an aortic damage during an exploratory laparoscopy due to a veress needle, which was followed by the development of AAP after 9 days and was successfully managed by open surgery. To our knowledge, this is the first example of AAP in which the injury site and the progression of AAP were distinct. A follow-up CT scan could be crucial in diagnosing AAPs, especially in patients who are asymptomatic. AAPs necessitate a quick surgical repair to avoid fatal consequences.

Keywords: Abdominal aortic pseudoaneurysm, Accidental injury, Veress needle injury, Trauma

\section{INTRODUCTION}

The abdominal aortic aneurysm (AAA) is defined as dilatation of minimum of $3 \mathrm{~mm}$ or expansion in the diameter greater than $50 \%$ of the original size which may involve any layers of the vessel. ${ }^{1}$ Depending upon the involvement of the layers, the type of the aneurysm is defined. Aneurysms can be defined as true if all three layers are involved, false or pseudo if the origin is of traumatic or infectious etiology (pseudoaneurysm) or dissecting aneurysm where there is a tear in the innermost layer of the aorta and a lumen is created in the middle of the wall of the vessel. ${ }^{2}$ The commonest location for such AAA is infrarenal. ${ }^{3}$

Pseudoaneurysms due to injury of the abdominal aorta are rare and often reported after either blunt trauma or penetrating vessels injury. ${ }^{4}$ The clinical presentation of abdominal aortic pseudoaneurysm (AAP) varies from asymptomatic to compression symptoms to fatal spontaneous rupture resulting in death.

We present a case of young female who bore an accidental aortic injury during abdominal access with veress needle during an hystero-laparoscopy which was followed by development of AAP after 9 days and successfully repaired by open surgery.

\section{CASE REPORT}

A 30 years old female, nulligravida, was admitted on $23 \mathrm{rd}$ July 2020 to the Mayflower hospital for the diagnostic hysteroscopy and laparoscopy for primary infertility and PCOD drilling. The patient was non diabetic and normotensive. Patient had history of migraine which was being managed by own by paracetamol SOS. Patient was a recently diagnosed hypothyroid.

Patient was prepared for the diagnostic laparoscopy after uneventful hysteroscopy under general anesthesia, pneumoperitoneum created with veress needle and the primary trocar entry was taken through umbilicus. The laparoscope introduced and laparoscopy revealed fresh blood in the pouch of Douglas and Morrison's pouch. Patient had tachycardia and blood pressure dropped and was non recordable. A vessel injury was suspected and the decision for conversion to laparotomy was taken.

Abdomen was opened by midline vertical incision extending from umbilicus up to pubic symphysis. All blood clots evacuated from the pouches of Douglas and 
Morrison, small intestine retracted packed with abdominal sponges. There was a large hematoma in the presacral region extending into the sigmoid mesocolon. Hematoma dissected and clots removed. There was jet of blood coming from the lower part of the abdominal aortic bifurcation. Bleeding site compressed with the abdominal sponges and kept in place till blood pressure improved and blood sample send for grouping and cross matching. Once blood pressure stabilised and blood products available, abdominal sponges removed, mesocolic hematoma evacuated and bifurcation of the aorta exposed, inferior mesenteric artery retracted laterally on left side, bleeding site identified at the aortic bifurcation and closed with prolene 3/0 interrupted sutures. Hemostasis was secured with no active bleeding. PCOD drilling was done and small myoma was excised along with left ovarian chocolate cyst. Drain placed in the pelvis and the abdominal cavity was closed and patient was shifted to the ward. Postoperative period was uneventful patient resumed normal diet on 3/4th postoperative day. Drain removed on 5th postop day. Patient had nonspecific complaint on 6/7th postoperative day so decision was taken to do CECT scan with angiography. To everyone's surprise there was a pseudoaneurysm of $1 \times 1 \times 1.7 \mathrm{~cm}$ arising from bifurcation of aorta and anterior wall of proximal left common iliac artery.

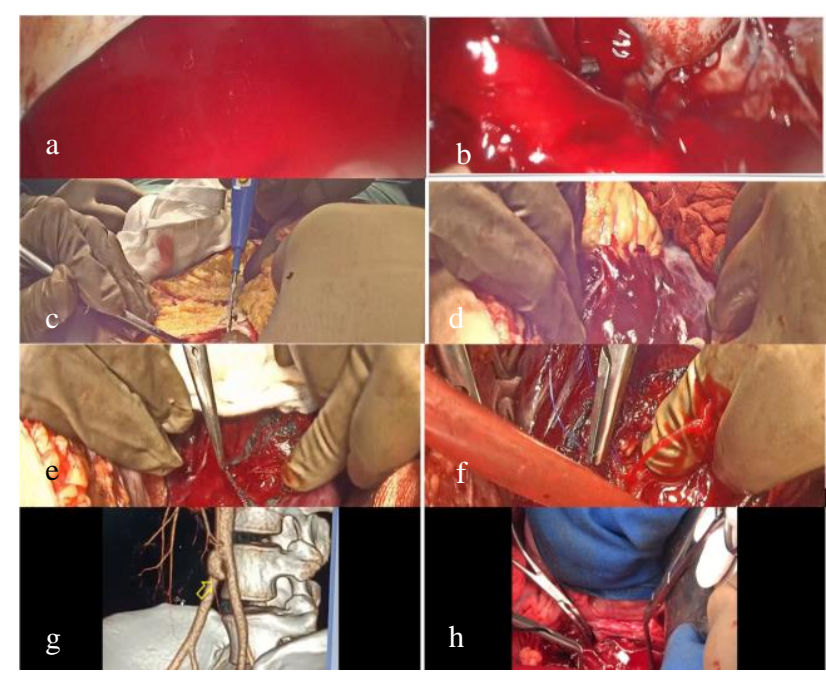

Figure 1: (a) Laparoscopy revealed a profusely good field with fresh blood clots in Morrisons pouch; (b) laparoscopy revealed a profusely good field with fresh blood clots in pouch of Douglas; (c) convert the surgery to a laparotomy and immediately opened the abdomen; (d) a huge hematoma was formed in the retroperitoneal region, at the site of bifurcation of aorta over the sacral promontory; (e) palpate the vessel by feeling its pulsations and then open the hematoma layer by layer; (f) finally reaching the actual site of injury and we can see bulging happen at the point of rupture. We take a big stitch with vicryl 3-0 followed by figure of eight stitch;

(g) on 6th day went in for a CT angiography, pseudoaneurysm of aorta found at the bifurcation of aorta;

(h) Pseudo-aneurysm of aorta excised and sutured with prolene 5-0 with teflon.

Link for procedure: https://www.youtube.com/watch?v= RjLMaEri8tk.
Patient and her family was explained about findings and treatment options were discussed in detail. Vascular surgeon's and intervention radiologist's consulted and a decision was taken for open repair of the pseudoaneurysm. The abdomen was opened through the same incision, bowels retracted, aorta exposed, proximal and distal vascular control taken with vascular slings and vascular clamps. After systemic heparinization, pseudoaneurysm sac opened, opening in the arterial wall identified and closed with interrupted, 5/0 pledgeted prolene. Distal and proximal clamps removed, hemostasis checked, and abdomen closure done with loop nylon. Postoperative period was uneventful and the patient was discharged on the 7 th postoperative day.

\section{DISCUSSION}

Penetrating abdominal aortic injuries occurs in less than $2 \%$ of the population and it is associated with very high mortality of up to $80 \% .^{5,6}$ Abdominal aortic pseudoaneurysm is a rare phenomenon which is observed after penetrating aortic injuries as well as blunt trauma. ${ }^{7}$ There is a possibility of disruption of the aortic wall integrity due to various factors like infection, inflammation or direct injury. This leads to development of a potential weakness in the wall which is then unable to sustain the aortic blood pressure. Resultantly, blood dissects in the layers of the aortic wall and forms a dilated sac covered by either media or adventitia of the aortic wall. This pseudoaneurysm differs from true aneurysms of aorta in terms of the layers of the aneurysm sac. All the three arterial wall layers are involved in case of true aneurysm.

The time duration for the formation of AAP after an injury is not known till now and may range from weeks to years. ${ }^{8}$ Any breach in the integrity of the aortic wall increases the possibilities of blood dissecting the wall, leading to blood collection under either medial or adventitia. This collected blood either remains at the site of injury which exerts the tamponade effect at the injury or may travel down to the gravity and gets collected in the lower parts around the bifurcation of aorta.

Any such AAP may remain asymptomatic for years and found accidently during routine investigation or sudden rupture. ${ }^{9}$ Many times it may also present as palpable mass, may surround blood vessels and cause compressive symptoms or acute compression of aorta due to compression. $^{10}$

For the treatment of such pseudoaneurysms, various options like open surgical repair, endovascular repair, thrombosis of the aneurysmal sac or coil embolization are available. For the open repair of such pseudoaneurysm, after opening up of the sac either resection and graft interposition or direct suturing is preferred. ${ }^{11,12}$

In a case reported published, an AAP of suprarenal location was associated with epigastric pain as well as compression of common bile duct leading to jaundice. The patient was operated and the repair was carried out by intraluminal patch aortoplasty.4 Multiple authors have reported their experience of endovascular repair of AAP 
by the application of stent graft as well as endoprosthesis. ${ }^{13-15}$

A case report of a young male with the history of upper GI bleeding and massive intraabdominal bleed was reported with the history of aortic perforation. The patient was diagnosed to have AAP through a CT scan after the surgical repairs of the intestinal as well as aortic injury. AAP was treated using balloon expandable bifurcated endoprosthesis. ${ }^{16}$

In other case reports, the ruptured AAP was associated with pancreatitis. This emergency was managed using aortomonoiliac endograft, which allowed immediate control on the hemorrhage in a potentially infective operative field. ${ }^{15}$

A case of AAP developing after a trauma 8 months back due to stab injury in the abdomen was reported by Oner et al. The location of AAP was near iliac bifurcation which was closed using bifurcated endovascular stent and the TriVascular Ovation endovascular stent. ${ }^{17}$

There have been reports of open surgical repair of AAPs. A case of an old man with his history of a large AAP following a surgical fenestration and patch aortoplasty was reported by Geckeis et al the patient was delivered transcatheter 1500 thrombin units which resulted in thrombosis of the sac. ${ }^{11}$

Our patient presented with accidental injury to abdominal aorta near bifurcation during an exploratory laparoscopy due to veress needle injury. The injury site of the aorta was immediately secured by open laparotomy and hemostasis secured. On 8th postop day of the repair, CECT examination showed an aortic pseudoaneurysm below the site of the previous injury. It is assumed that there is some leak of blood occurred from primary repair site which resulted in the pseudoaneuryam formation. We opted for the open repair of the pseudoaneurysm because of the location of the pseudoaneurysm which required Y shaped stent graft, very high cost of the endovascular treatment, and young age of the patient with relatively small caliber aorta.

\section{CONCLUSION}

Iatrogenic abdominal aortic injury is a nightmare for any laparoscopic surgeons as it warrants immediate intervention to save the patient's life. The magnitude of stress goes exponentially high when it occur in a patient who is planned for surgery of lesser magnitude. In this case we were fortunate that timely intervention could save the patient. would like to stress Abdominal aortic pseudoaneurysms after injury are quite rare and may remain asymptomatic for weeks to months. This may result in to fatal haemorrhage leading to death. A follow up CT scan might prove to be of great importance in diagnosing AAPs, particularly in asymptomatic patients.
AAPs warrant a prompt intervention in order to avoid the fatal complications.

Funding: No funding sources

Conflict of interest: None declared

Ethical approval: Not required

\section{REFERENCES}

1. Wanhainen A. How to define an abdominal aortic aneurysm--influence on epidemiology and clinical practice. Scand J Surg. 2008;97:105-9.

2. Kent KC. Clinical practice. Abdominal aortic aneurysms. N Engl J Med. 2014;371:2101-8.

3. Sakalihasan N, Limet R, Defawe OD. Abdominal aortic aneurysm. Lancet. 2005;365:1577-89.

4. Chase CW, Layman TS, Barker DE. Traumatic abdominal aortic pseudoaneurysm causing biliary obstruction: a case report and review of the literature. J Vasc Surg. 1997;25:936-40.

5. Demetriades D, Theodorou D, Murray J. Mortality and prognostic factors in penetrating injuries of the aorta. $\mathrm{J}$ Trauma. 1996;40:761-3.

6. Parmley LF, Mattingly TW, Manion WC. Penetrating wounds of the heart and aorta. Circulation. 1958;17:953-73.

7. Ishii H, Nakamura K, Nakamura E. Aortic pseudoaneurysm due to simple vertebral compression fracture treated with conservative management. Ann Vasc Dis. 2016;9:349-51.

8. Massara M, Prunella R, Gerardi P. Infrarenal Abdominal Aortic Pseudoaneurysm: Is It a Real Emergency?. Ann Vasc Dis. 2017;10(4):423-5.

9. Borioni R, Garofalo M, Seddio F, Colagrande L, Marino B, Albano P. Posttraumatic infrarenal abdominal aortic pseudoaneurysm. Tex Heart Inst J. 1999;26(4):312-4.

10. Li X, Zhao JC, Huang B. Management of giant posttraumatic abdominal aortic pseudoaneurysm and aortic occlusion using a unique hybrid procedure combining transcatheter device closure and open surgical repair. Ann Vasc Surg. 2014;28:1322.e7-11.

11. Geckeis K, Eggebrecht H, Schmermund A. Percutaneous repair of abdominal aortic pseudoaneurysm by catheterbased delivery of thrombin. J Endovasc Ther. 2006;13:2648.

12. Salsamendi J, Pereira K, Rey J. Endovascular coil embolization in the treatment of a rare case of posttraumatic abdominal aortic pseudoaneurysm: brief report and review of literature. Ann Vasc Surg. 2016;30:310.e1-8.

13. Ghazala CG, Green BR, Williams R. Endovascular management of a penetrating abdominal aortic injury. Ann Vasc Surg. 2014;28:1790.e9.

14. Zamudio L, Olive G, Barone H. Traumatic abdominal aortic pseudoaneurysm treated with balloon-expandable bifurcated endoprosthesis. J Vasc Surg. 1998;28:345-8.

15. Hinchliffe RJ, Yung M, Hopkinson BR. Endovascular exclusion of a ruptured pseudoaneurysm of the infrarenal abdominal aorta secondary to pancreatitis. J Endovasc Ther. 2002;9:590-2.

16. Bechara-Zamudio L, Olive G, Barone H. Traumatic abdominal aortic pseudoaneurysm treated with balloonexpandable bifurcated endoprosthesis. J Vasc Surg. 1998;28:345-8.

17. Oner E, Erkanli K, Akturk IF. Posttraumatic infrarenal abdominal aortic pseudoaneurysm treated with bifurcated endovascular graft stent. Postepy Kardiol Interwencyjnej. 2015;11:146-9.

Cite this article as: Patel S, Patel S, Khiria L.

Traumatic pseudoaneurysm of aorta: a story of survival. Int J Reprod Contracept Obstet Gynecol 2022;11:944-6. 